\title{
Preseason to Postseason Changes on the BTrackS Force Plate in a Sample of College Athletes
}

\author{
Ryan Morrison, Kyle M. Petit, Chris Kuenze, Ryan N. Moran, and Tracey Covassin
}

\begin{abstract}
Context: Balance testing is a vital component in the evaluation and management of sport-related concussion. Few studies have examined the use of objective, low-cost, force-plate balance systems and changes in balance after a competitive season. Objective: To examine the extent of preseason versus postseason static balance changes using the Balance Tracking System (BTrackS) force plate in college athletes. Design: Pretest, posttest design. Setting: Athletic training facility. Participants: A total of 47 healthy, Division-I student-athletes (33 males and 14 females; age 18.4 [0.5] y, height 71.8 [10.8] cm, weight 85.6 [21.7] kg) participated in this study. Main Outcome Measures: Total center of pressure path length was measured preseason and postseason using the BTrackS force plate. A Wilcoxon signed-rank test was conducted to examine preseason and postseason changes. SEM and minimal detectable change were also calculated. Results: There was a significant difference in center of pressure path length differed between preseason $(24.6[6.8] \mathrm{cm})$ and postseason $(22.7[5.4] \mathrm{cm})$ intervals $(P=.03)$, with an SEM of $3.8 \mathrm{~cm}$ and minimal detectable change of $10.5 \mathrm{~cm}$. Conclusions: Significant improvements occurred for center of pressure path length after a competitive season, when assessed using the BTrackS in a sample of college athletes. Further research is warranted to determine the effectiveness of the BTrackS as a reliable, low-cost alternative to force-plate balance systems. In addition, clinicians may need to update baseline balance assessments more frequently to account for improvements.
\end{abstract}

Keywords: balance, concussion, postural sway, reliability

The most commonly reported symptoms following a sportrelated concussion (SRC) are headaches, dizziness, and balance problems. ${ }^{1}$ Best practices for SRC assessment include symptom evaluation, neuropsychological testing, vestibular and ocular motor testing, and balance assessment. ${ }^{1}$ The National Collegiate Athletic Association now mandates preparticipation baseline SRC assessments be completed for varsity student-athletes and must include a balance component. ${ }^{2}$ Institutions may utilize any balance measure that best fits their needs as long as objective data are collected for. ${ }^{2}$

Balance can be measured using a variety of assessments, including the balance error scoring system, clinical test of sensory integration of balance, and Romberg's test. The balance error scoring system test has been shown to be a valid assessment for acute SRCs, with low sensitivity (0.34) and high specificity (0.96). ${ }^{3,4}$ In addition, the Romberg's test has moderate sensitivity (0.55) and specificity (0.64) for individuals with vestibular dysfunction. ${ }^{5}$ One concern with these tests is their subjective scoring, as this is done via a rater using preestablished criteria. This may result in issues regarding diagnostic accuracy and reliability. Due to the subjective nature of these tests, a force plate is considered the gold standard because of its objective outcome. ${ }^{6}$ Force-plate analysis allows for the athlete center of pressure (COP) to be calculated based on the foot contact of an individual while on the plate. ${ }^{7}$ Although use of a force plate is the preferred method, its high cost and technical demands make it impractical for many clinicians. ${ }^{7}$ This has led to the development of low-cost alternatives similar to that of research-grade force platforms.

Morrison is with the Athletic Department, University of Maryland, College Park, MD. Petit, Kuenze, and Covassin are with Sports Injury Research Lab, Department of Kinesiology, Michigan State University, East Lansing, MI, USA. Moran is with Athletic Training Research Lab, Department of Health Science, The University of Alabama, Tuscaloosa, AL, USA. Petit (petitkyl@msu.edu) is corresponding author.
The Balance Tracking System (BTrackS) is a portable force plate that provides an objective balance assessment via estimation of COP path length. ${ }^{7}$ Preliminary investigation of the BTrackS has yielded a specificity of 0.90 in concussed college athletes. ${ }^{8}$ The BTrackS has also displayed good-to-excellent test-retest reliability when participants were tested at ranges up to 15 days. $^{9}$ Similarly, an intraclass correlation coefficient $\left(\mathrm{ICC}_{2,1}\right)$ of .92 was found for the BTrackS, indicating excellent test-retest reliability. ${ }^{9}$ Research has yet to examine the effects of a competitive athletic season on BTrackS outcomes.

Balance assessments have been criticized because of their potential to be influenced by strength improvements, sport-related activities, and proprioceptive training, which are common during athletic participation. Previous research has demonstrated balance improvements after various training protocols, furthering the idea that balance may be influenced through sport training. ${ }^{10}$ Specifically, researchers found balance improvements on the balance error scoring system and star excursion balance test after just a 6-week neuromuscular training program. ${ }^{11}$ The program, employed by researchers, consisted of common training techniques often found throughout an athletic season. These balance changes achieved during an athletic season suggest a possible threat to preseason assessments, further making postconcussion balance deficits harder to identify. Therefore, the purpose of this study was to examine preseason versus postseason changes in COP path length using the BTrackS force plate in college student-athletes. It was hypothesized that BTrackS performance would improve over an athletic season when compared with baseline measures.

\section{Methods}

\section{Design}

The current study utilized a pretest, posttest design. The dependent variable was COP path length (in centimeters). COP path length is 
a proxy for total body sway, with larger values indicating worse performance. The independent variable was time spanning from preseason to postseason. The study was approved by the Michigan State University Institutional Review Board, and all participants provided informed consent before testing.

\section{Participants}

A total of 47 healthy college student-athletes, between 18 and 22 years old (33 males and 14 females; age 18.4 [0.5] y, height 71.8 [10.8] cm, weight 85.6 [21.7] $\mathrm{kg}$ ) participated in this study. Participants were recruited from a Division-I university athletic program. Participants represented 5 sports, with the majority competing in football $(\mathrm{n}=28,59.6 \%)$ and women's soccer $(\mathrm{n}=8$, $17.0 \%$ ). Additional sport breakdown can be found in Table 1. Exclusion criteria consisted of student-athletes who were not participating in a fall season sport or who had currently suffered a concussion or lower-extremity injury.

\section{Instrumentation}

Center of pressure path length was measured using the BTrackS (Balance Tracking Systems Inc, San Diego, CA) force plate. COP path length was defined as the total distance (in centimeters) traveled by the $X-Y$ trajectory of the COP during a 20 -second trial. BTrackS has also been found to have excellent test-retest reliability $(\mathrm{ICC}=.92){ }^{9}$

\section{Procedures}

Participants reported to the university research laboratory or athletic training facility to complete the baseline balance testing prior to their competitive season. Participants stood on the force plate, with their feet shoulder width apart, their eyes closed, and their hands on their hips for a 20-second trial. Participants completed 3 trials on the BTrackS, with a 30 -second break between trials. The mean of the 3 trials represented participant's COP path length for that test interval (preseason or postseason). After the competitive season, participants completed the same testing protocol.

\section{Table 1 Participants' General Characteristics $(\mathrm{N}=47)$}

\begin{tabular}{lc}
\hline Demographic & $\mathbf{n}(\%)$ \\
\hline Sex & \\
Male & $33(70.2)$ \\
Female & $14(29.8)$ \\
Sport & \\
Football & $28(59.6)$ \\
Women's soccer & $8(17.0)$ \\
Men's soccer & $5(10.6)$ \\
Women's volleyball & $3(6.4)$ \\
Field hockey & $3(6.4)$ \\
\hline
\end{tabular}

\section{Statistical Analyses}

Data were summarized using mean and SD or median and interquartile range. Results from a Shapiro-Wilk test revealed that the data were not normally distributed; thus, a Wilcoxon signed-rank test was conducted to assess preseason versus postseason COP differences. The SEM was calculated as $\mathrm{SD} \times \sqrt{ } 1-\mathrm{ICC},{ }^{12}$ and the minimal detectable change (MDC) was calculated as $1.96 \times$ $\mathrm{SEM} \times \sqrt{ } 2 .{ }^{13}$ The $\mathrm{SEM}$ is defined as the estimate of the variability between the score obtained and the participants "true" score. ${ }^{12}$ The MDC reflects the smallest change in score that can be interpreted as a real and not from measurement error. ${ }^{13}$ All data were analyzed using SPSS (version 24; IBM Corp, Armonk, NY), and statistical significance was set a prior at $P<.05$.

\section{Results}

Center of pressure path length was found to be significantly different between preseason and postseason balance testing $(Z=$ $-2.13, P=.03$ ). This study identified an SEM of 3.8 and an MDC of $10.5 \mathrm{~cm}$ for the BTrackS (Table 2). Differences between individual preseason and postseason scores ranged from 0.0 to $14.0 \mathrm{~cm}$, with $30(63.8 \%)$ participants improving, $16(34.0 \%)$ worsening, and 1 participant scoring the same between testing periods. Roughly half $(\mathrm{n}=24,51.1 \%)$ of student-athletes displayed a change in COP greater than the SEM, and $4(8.5 \%)$ displayed a change that exceeded the MDC.

\section{Discussion}

The current study examined the extent of preseason versus postseason changes in COP path length as measured by the BTrackS force plate in college student-athletes. The BTrackS has been found to be a reliable balance assessment during serial testing. ${ }^{9}$ Our findings indicate that there were statistically significant differences between preseason and postseason COP path length, suggesting balance improvements after an athletic season.

Despite finding statistically significant improvements in COP path length after a single athletic season, only half of participants experienced a change that exceeded the SEM for the BTrackS. Furthermore, only 4 student-athletes experienced a change that exceeded the MDC $(10.46 \mathrm{~cm})$, which indicates a clinically meaningful change. These results suggest that although improvements in COP after a single athletic season may exist, several athletes will not experience a change large enough to exceed the measurement error of the BTrackS. The COP changes noticed in the current results are similar to those of Heleno et $\mathrm{al}^{10}$ who reported significant balance improvements after a 5-week training program. Balance improvements have also been reported in soccer athletes following certain tasks, such as a dynamic warm-up. ${ }^{14}$ These results may be further supported by Thompson et al ${ }^{15}$ who identified greater balance scores in athletes compared with nonathletes. These balance changes, along with those of the current study, suggest that

Table 2 BTrackS Preseason and Postseason Scores in NCAA Student-Athletes

\begin{tabular}{lccccc}
\hline & Mean (SD) & Median (IQR) & Range & $\boldsymbol{P}$ value & SEM \\
\hline Preseason & $24.6(6.8)$ & $22.0(8.0)$ & $11-41$ & .03 & 3.8 \\
Postseason & $22.7(5.4)$ & $21.0(7.0)$ & $14-38$ & & \\
\hline
\end{tabular}

Abbreviations: IQR, interquartile range; MDC, minimal detectable change; NCAA, National Collegiate Athletic Association. 
even a single athletic season may impact baseline balance assessments. Therefore, it may be necessary to readminister COP assessments more often than once per athletic career to account for any improvements made throughout a single season.

The current study had some limitations. First, it included a small number of Division-I student-athletes from a small array of sports. In addition, data were collected over 1 athletic season, leaving little comparison with 1-year or 2-year test-retest interval. The data were also collected at the end of each sport, with some student-athletes playing more games than others. Another limitation was not accounting for injuries that may have occurred throughout the athletic season. Early season injuries, such as ankle sprains, may have impacted BTrackS performance due to lingering proprioceptive impairments. Furthermore, COP improvements could not solely be contributed to physiological balance improvements, but may be in combination with BTrackS measurement error.

Future research should aim to include equal male and female student-athletes, with a larger sample over a longer period. Observing baseline changes over a college career would give clinicians a better understanding of how often to collect baseline data, as there are no consistent recommendations. Identifying which strength and conditioning programs are used by each sport should also be a focus, as they may influence balance. Additional balance assessment tools (ie, sway balance) should be explored for their clinical utility for evaluating balance deficits.

\section{Conclusions}

The results of this study found significant differences on BTrackS COP path length between preseason and postseason time intervals; however, only half of the participants exceeded the SEM of BTrackS. This suggests that clinicians may need to consider updating baseline balance assessments more frequently than once per athletic career. Furthermore, an outdated baseline assessment may complicate postconcussion balance testing.

\section{References}

1. McCrory P, Meeuwisse W, Dvorak J, et al. Consensus statement on concussion in sport - the 5th international conference on concussion in sport held in Berlin, October 2016. Br J Sports Med. 2017; 51(11):838-847. PubMed ID: 28446457

2. NCAA. Concussion Guidelines: Diagnosis and Management of Sport-Related Concussions. 2014. http://www.ncaa.org/health-andsafety/concussion-guidelines. Accessed November 11, 2017.

3. McCrea M, Guskiewicz KM, Marshall SW, et al. Acute effects and recovery time following concussion in collegiate football players: the NCAA concussion study. JAMA. 2003;290(19):2556-2563. PubMed ID: 14625332 doi:10.1001/jama.290.19.2556
4. Riemann BL, Guskiewicz KM. Effects of mild head injury on postural stability as measured through clinical balance testing. $J$ Athl Train. 2000;35(1):19-25. PubMed ID: 16558603

5. Jacobson GP, McCaslin DL, Piker EG, Gruenwald J, Grantham S, Tegel L. Insensitivity of the "Romberg test of standing balance on firm and compliant support surfaces" to the results of caloric and VEMP tests. Ear Hear. 2011;32(6):e1-e5. PubMed ID: 21775891 doi:10.1097/AUD.0b013e31822802bb

6. Chang JO, Levy SS, Seay SW, Goble DJ. An alternative to the balance error scoring system: using a low-cost balance board to improve the validity/reliability of sports-related concussion balance testing. Clin J Sport Med. 2014;24(3):256-262. PubMed ID: 24284947 doi:10.1097/JSM.0000000000000016

7. O'Connor SM, Baweja HS, Goble DJ. Validating the BTrackS balance plate as a low cost alternative for the measurement of sway-induced center of pressure. J Biomech. 2016;49(16):41424145. doi:10.1016/j.jbiomech.2016.10.020

8. Goble DJ, Manyak KA, Abdenour TE, Rauh MJ, Baweja HS. An initial evaluation of the BTrackS balance plate and sports balance software for concussion diagnosis. Int J Sports Phys Ther. 2016; 11(2):149-155. PubMed ID: 27104048

9. Hearn MC, Levy SS, Baweja HS, Goble DJ. BTrackS balance test for concussion management is resistant to practice effects. Clin J Sport Med. 2018;28(2):177-179. PubMed ID: 28452829

10. Heleno LR, da Silva RA, Shigaki L, et al. Five-week sensory motor training program improves functional performance and postural control in young male soccer players-a blind randomized clinical trial. Phys Ther Sport. 2016;22:74-80. PubMed ID: 27620862 doi:10. 1016/j.ptsp.2016.05.004

11. McLeod TC, Armstrong T, Miller M, Sauers JL. Balance improvements in female high school basketball players after a 6-week neuromuscular-training program. J Sport Rehabil. 2009;18(4):465481. PubMed ID: 20108849 doi:10.1123/jsr.18.4.465

12. McManus IC. The misinterpretation of the standard error of measurement in medical education: a primer on the problems, pitfalls and peculiarities of the three different standard errors of measurement. Med Teach. 2012;34(7):569-576. PubMed ID: 22509894 doi:10. 3109/0142159X.2012.670318

13. de Vet HC, Terwee CB, Knol DL, Bouter LM. When to use agreement versus reliability measures. J Clin Epidemiol. 2006; 59(10):1033-1039. PubMed ID: 16980142 doi:10.1016/j.jclinepi. 2005.10.015

14. Daneshjoo A, Mokhtar AH, Rahnama N, Yusof A. The effects of comprehensive warm-up programs on proprioception, static and dynamic balance on male soccer players. PLoS ONE. 2012;7(12): e51568. PubMed ID: 23251579 doi:10.1371/journal.pone.0051568

15. Thompson LA, Badache M, Cale S, Behera L, Zhang N. Balance performance as observed by center-of-pressure parameter characteristics in male soccer athletes and non-athletes. Sports. 2017;5(4):E86. doi: $10.3390 /$ sports5040086 